\title{
Análisis histórico de la reforma psiquiátrica andaluza: la salud mental reformada sin manicomios
}

\author{
José Carmona Calvo \\ Francisco del Río Noriega \\ Servicio Andaluz de Salud
}

\begin{abstract}
Resumen
Se expone en las páginas siguientes el proceso de transformación de los recursos psiquiátricos habido en la región de Andalucía, España. En esta segunda parte se analiza el desarrollo acaecido después de la desaparición del organismo creado para llevar a cabo la reforma. Así, se ofrece una visión crítica de la integración de los recursos de salud mental en el sistema sanitario general, del desmantelamiento completo de los hospitales psiquiátricos, de la creación de servicios sociales específicos para personas con trastornos mentales graves y de la situación actual de la salud mental "reformada", dando cuenta de los logros y de las dificultades de cada etapa de este extenso periodo de veinte años:1991-2011.
\end{abstract}

Palabras claves: reforma psiquiátrica; salud mental, hospital psiquiátrico; trastorno mental

\begin{abstract}
Historical analysis of the psychiatric reform in Andalucia: mental health reform without asylums. The following paper talks about the transformation process of psychiatrics resources in Andalucia, Spain. This second part analyzes the development that took place after the disappearance of the organism created to carry out the reform. It offers a critical approach to the incorporation of the mental health resources into the general sanitary system, to the complete extinction of the psychiatric hospitals, to the creation of specific social services for people suffering from serious mental disorder and to the present situation of the "reformed" mental health, informing about the goals and difficulties of each phase of these long twenty years: 1991-2011.
\end{abstract}

Keywords: psychiatric reform; mental health; psychiatric hospitals; serious mental disorder.

$\mathrm{T}$ ras casi tres décadas después de la creación del Instituto Andaluz de Salud Mental (IASAM), el camino recorrido ha sido arduo y complejo y, como todos los procesos de grandes transformaciones, con sus luces y sus sombras. Puede decirse que después de la desaparición del IASAM, como corresponde después de una época llena de compromiso y entusiasmo, vino el desencanto. La no consecución, a corto plazo, de los resultados deseados puede que sea una de las causas del mismo, pero también a aparición de situaciones nuevas con problemáticas difíciles de resolver. Al fin y al cabo, lo que puso de manifiesto la reforma de los primeros años eran las grandes carencias que tenía la atención a la salud mental. Pero a medida que fueron creándose recursos, no siempre al ritmo deseado, y fueron incorporándose nuevos profesionales que comenzaron a conformar redes cada vez más complejas, el ajuste y adaptación de todo el proceso requería tiempo. Quizás en los últimos años ha confluido la sedimentación de experiencias profundas de transformación, junto con interés renovado por la calidad de los servicios desde la Administración Sanitaria. Esquemáticamente la década de los noventa fue la del desencanto y la primera de este siglo la revitalización de los procesos de transformación. Sin embargo hay que decir que no todo fue desencanto en los noventa, ni todo es progreso en la última década. Pretendemos ofrecer un análisis histórico algo más riguroso, más allá de los reduccionismos y esquematismos.

\section{Etapa post-IASAM: 1991-2000}

Se ha identificado siempre esta etapa como de recesión general de todo el proceso de reforma. Y aunque esto ha sido así, en términos generales, también ocurrieron eventos importantes para la culminación del proceso de reforma, para el desarrollo de los servicios sanitarios y para la integración de los servicios administrativos del IASAM en los servicios centrales del SAS. Concretamente esta integración ha necesitado toda una década para comenzar a comprender el proceso complejo que significaba. Pero, a pesar de ello, en esta década que analizamos, tuvo lugar el cierre efectivo de los manicomios, prueba evidente de que, a pesar de la parálisis señalada, el proceso se había hecho irreversible. En esa misma línea tiene lugar la puesta en marcha de las primeras comunidades terapéuticas, aunque hay que decir que se implantan de forma mínima este tipo de dispositivos.

Desde otra instancia, el Parlamento de Andalucía, se produce la creación de la Fundación Andaluza para la Integración Social del Enfermo Mental (FAISEM), organismo encargado de 
establecer los servicios sociales específicos que los pacientes, con trastorno mental grave, requieren como complemento a los recursos sanitarios para vivir en la comunidad. Por último, se originan, o por lo menos, toman mucha mayor fuerza los movimientos asociativos de usuarios y allegados, que juntamente con los profesionales, unas veces juntos y otras por separado, suponen un contrapunto a la apatía que el sistema sanitario general demostraba con la salud mental, tanto, que su presión va a ser determinante en la reanimación del proceso en la década siguiente. Todo este proceso, complejo y contradictorio, conviene analizarlo de forma pormenorizada, como exponente de una década de sombras y luces, muy contrastada y criticada.

\section{La creación de FAISEM. Análisis. Datos de desar- rollo.}

En diciembre de 1993 se creó la Fundación Andaluza para la Integración Social del Enfermo Mental (FAISEM), por iniciativa del Parlamento de Andalucía. Por unanimidad de todos los grupos políticos se instaba al gobierno a la creación de un organismo público que garantizase la integración social y laboral de personas gravemente discapacitadas por la enfermedad mental. Es de destacar la unanimidad, pues fue la única disposición parlamentaria que la lograba ese año, fiel reflejo de la necesidad sentida por la sociedad en general, y los movimientos asociativos de familiares y profesionales, en particular, de garantizar esa cobertura social a personas que, debido a la enfermedad, habían perdido una autonomía personal y funcional para la vida en comunidad.

La FAISEM tiene como misión el desarrollo y gestión de recursos de apoyo social para pacientes que sean usuarios de los servicios públicos de salud mental con el objetivo general de la prevención de la marginación y la desadaptación de cualquier persona afectada por una enfermedad mental grave, causante de una incapacidad personal y social, así como el apoyo a su integración social y laboral ${ }^{1}$. La puesta en marcha de la FAISEM, a pesar de su escasez de recursos y de presupuestos al principio de su andadura, marcó un hito importante en el desarrollo de la Reforma Psiquiátrica, aportando un apoyo imprescindible en el tratamiento, en el pronóstico y en la calidad de vida de los ciudadanos que padecen un Trastorno Mental Grave y de sus familiares.

Como sabemos, las necesidades que requieren las personas con trastorno mental grave para la convivencia en la comunidad, son de muy variada índole: alojamiento, reeducación en capacidades básicas, interpersonales y sociales, poder garantizar la ocupación y promoción al empleo, ayudas sociales, poder disfrutar del ocio y del tiempo libre, educación académica, etc (Torres González, 1999). Algunas de ellas deben estar cubiertas por el sistema social normalizado; pero cuando esto no sea posible deben existir unos servicios sociales específicos. Dichos servicios son los que se inician en 1993 de la mano de FAISEM, cuando a estas alturas ya se tiene claro que los servicios sociales generales no van a dar respuesta a las necesidades mencionadas y que, por la parte sanitaria, la reforma no solo requiere equipos comunitarios y unidades de corta hospitalización.

\section{Organigrama de FAISEM}

El organigrama de FAISEM de la figura 1 ofrece una estructura que refleja lo anteriormente dicho. Es decir, se trata de una fundación pública regida por un patronato constituido por las cuatro consejerías con competencias relacionadas con las necesidades mencionadas. La gestión se lleva a cabo por una gerencia, con sus órganos centrales y las respectivas delegaciones provinciales. Para la gestión específica de las empresas sociales (una por provincia andaluza), se creó IDEA (Iniciativa de Empleo Andaluza), directamente dependiente de la dirección gerencia y que, a modo de matriz, dirige las ocho empresas provinciales, que tienen gestión independiente. Hay que destacar que todas las empresas son sociedades anónimas, lo que indica su participación en el mercado libre, pero por otro lado, mantienen su carácter no lucrativo, como corresponden a centros especiales de empleo, donde todos los beneficios son reinvertidos en el propio desarrollo de las mismas. Existen, por otra parte, dos órganos asesores - la Comisión Asesora y el Comité de Expertos - con participación no solo de técnicos de las consejerías implicadas, sino también de asociaciones de afectados y familiares y también de personas pertenecientes a organizaciones que intervienen en los distintos programas de atención.

Los principales programas que gestiona FAISEM son: Programa Residencial, Programa Ocupacional- FormativoLaboral, Fomento de Relaciones Sociales, Promoción y Apoyo a entidades Tutelares, Evaluación, investigación, formación y cooperación internacional, y Apoyo al Movimiento Asociativo.

Es importante resaltar que el modelo de gestión de estos programas, salvo el residencial, procura la implicación, mediante convenios, de las más diversas organizaciones: administraciones locales, diversas ONGs y movimiento asociativo. Lo interesante de este modelo es la solidez que adquiere al comprometer en la gestión a organizaciones de diversos ámbitos, haciendo cada vez más tupida la red de atención, lo que a su vez supone una herramienta en la lucha contra el estigma de la enfermedad mental.

Si lo anterior supone un elemento estratégico a largo plazo, en el corto plazo supuso una serie de desencuentros entre las distintas entidades que comenzaron a trabajar en alguna de las facetas de la atención sociosanitaria. Y el más importante, sin duda, entre SAS y FAISEM, sobre todo en la década que analizamos. Las distintas administraciones tuvieron que hacer los reajustes necesarios para adaptarse a la evolución que la reforma tomaba en este período. Así, se alzaron voces desde los servicios de salud mental, hoy ya minoritarias, en contra de la creación de FAISEM; bien, en el sentido de que fuera la administración sanitaria la encargada de proveer los servicios específicos -los relacionados con alojamientos y talleres ocupacionales- para personas con trastornos graves; bien de que fuera la administración en general y, la sociedad en su conjunto, la que suministrara de servicios básicos educativos, sociales, laborales, etc., evitando, de esta forma, la marginación del enfermo mental, pues todo lo que fuese recursos específicos redundaría en la segregación.

Lo curioso de toda esta situación era estar moviéndose continuamente en una permanente paradoja: de un lado, había que avanzar en modelos de atención comunitaria, lo que suponía la entrada en escena de nuevos profesionales, no solo sanitarios, 


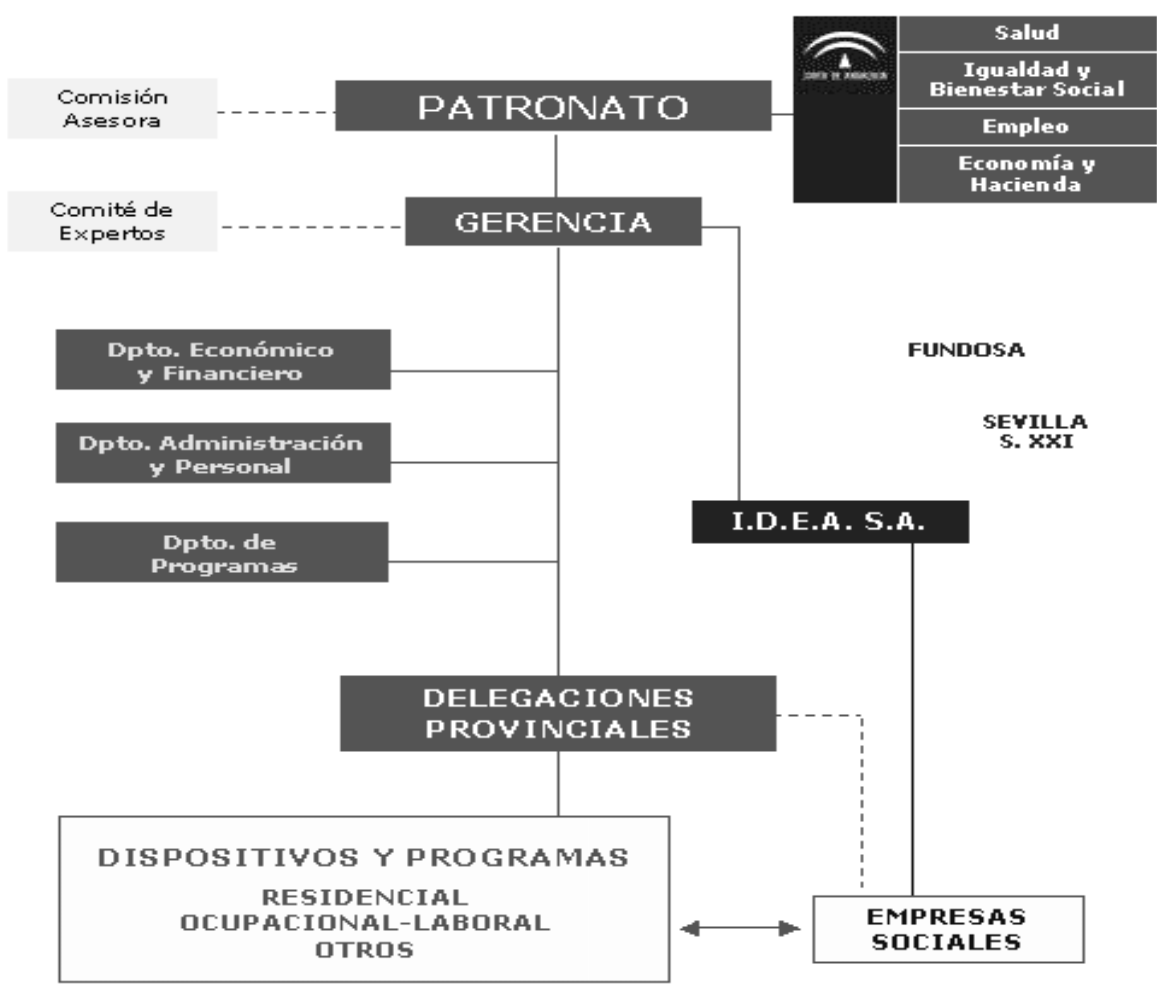

Figura 1. Organigrama de FAISEM

sino también de otras profesiones polivalentes que ofrecieran apoyo y soporte afectivo, social, laboral, etc, y, además de nuevas redes, sin exclusión de las generales ni de las específicas; de otro, el recelo a perder la hegemonía de la asistencia psiquiátrica tradicional. Sea como fuere, la implantación de FAISEM al final de la década fue un hecho irreversible, cuyas aportaciones cuantitativas no admiten discusión como puede verse en la siguiente tabla de evolución de los recursos sociales para los pacientes con trastorno mental grave (Tabla 1):

Desde el punto de vista cualitativo, podríamos resumir lo anteriormente expuesto en los siguientes resultados:

Tabla 1

Evolución recursos FAISEM 1984-2001

\begin{tabular}{llcc}
\hline Tipos de recursos & & 1984 & 2001 \\
\hline Recursos residenciales & Número & 9 & 75 \\
Pisos & Plazas & 39 & 267 \\
Casas-Hogar & Número & 0 & 17 \\
& Plazas & 0 & 268 \\
Residencias conveniadas & Número & 0 & 46 \\
& Plazas & 0 & 247 \\
Pensiones conveniadas & Número & 0 & 6 \\
& Plazas & 0 & 18 \\
Recursos ocupacionales-laborales & Número & 0 & 86 \\
Talleres Ocupacionales & Plazas & & 1.290 \\
& Número & 0 & 97 \\
Cursos de formación profesional & Plazas & & 522 \\
& Número & 0 & 8 \\
Empresas sociales & Plazas & 0 & 155 \\
\multirow{2}{*}{ Area de fomento de relaciones sociales } & & \\
Clubes sociales & Número & 1 & 17 \\
& Plazas & 30 & 510 \\
Plan de vacaciones & Plazas & & 945 \\
\hline Nor Fun &
\end{tabular}

Nota. Fuente: Programa de Salud Mental. Año 2001 enriquecimiento del trabajo comunitario, al disponer de otros soportes socio-laborales, que hasta la fecha no se disponían; como consecuencia, la ampliación de la red implica la cooperación intersectorial para garantizar la continuidad; función de bisagra entre servicios sociales comunitarios, especializados y específicos de salud mental; garantías de equidad en la provisión de recursos por acceso único desde el sistema de salud mental; y modelo de gestión participativa, no concertado, a diferencia de la atención social de deficientes, ancianos que no garantizaría lo anterior.

\section{El cierre de la red de manicomios. El proceso de desmantelamiento.Desaparición de los Hospitales Psiquiátricos}

El proceso de desmantelamiento - que tuvo una duración de 15 años, entre 1985 y 2000 - se inició ya desde el IASAM en una primera fase mediante el estricto control de los nuevos ingresos o reingresos de media y larga estancia y la búsqueda individualizada de otras alternativas. Paralelamente se realizó la división de los residentes de los ocho hospitales psiquiátricos en poblaciones diferenciadas: ancianos, casos estrictamente sociales, personas diagnosticadas de retraso mental, pacientes con sintomatología residual y pacientes con psicopatología activa, buscándose soluciones grupales o individuales (familias, o instituciones adecuadas, como las destinadas a deficientes, ancianos, etc.). De otro lado las comunidades terapéuticas que fueron poniéndose en marcha constituyeron el destino de la mayoría de los casos de pacientes con escasas o nulas habilidades psicosociales y/o psicopatología activa.

Gran parte de este proceso tuvo lugar en la etapa que ahora analizamos, probablemente porque la inercia acumulada desde 
el período anterior, lo hacía ya irreversible. No obstante, merece la pena detenernos un poco en él. La población asilada en los hospitales psiquiátricos venía ya disminuyendo desde 1977, año desde el que se comienzan a tener datos fiables en Andalucía, como ocurría por otra parte en el resto de las regiones de Europa (Freeman, Fryers, \& Henrderson, 1985). La tabla siguiente muestra la disminución de la población una vez iniciada la reforma en nuestra comunidad:

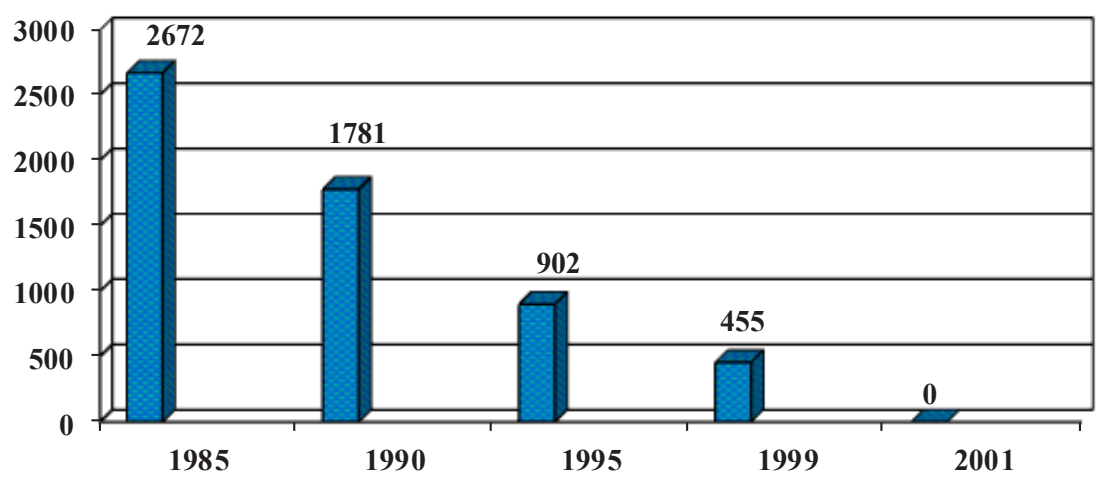

Figura 2. Población ingresada en los hospitales psiquiátricos

Como puede observarse, la cadencia de cierre de camas desde 1985 a 1999 es prácticamente simétrica cada cinco años. Al final de la década todavía quedaban 455 pacientes, cuya externalización resultaba más dificultosa, por cuanto se trataba de personas, en su mayoría, con diagnóstico de psicosis muy residuales, con gran deterioro y funcionalidad muy limitada, siendo muy escasas las estructuras para su acogimiento en la comunidad. Se llegó a un punto crítico en el que, o se finalizaba el cierre definitivo de las estructuras manicomiales, o se consolidaba para el futuro alguna(s) unidad(es) de larga estancia, eufemismo de nuevo manicomio, por lo que significaba de respuesta sociosanitaria única y cerrada a personas con discapacidad, y además, por la posibilidad de nuevos ingresos y el consiguiente riesgo de desandar el camino recorrido. A modo de resumen conviene hacer las siguientes reflexiones de todo este proceso:

La realidad de que se disponía de unos hospitales psiquiátricos generadores de conicidad y marginación $\mathrm{y}$, por tanto, de nula capacidad terapéutica.

La constatación de que es posible la asistencia psiquiátrica sin hospitales psiquiátricos, si se disponen de redes complejas de atención sociosanitaria.

La prevención, para el futuro, de la marginación al no disponer de estas estructuras.

La desaparición de los manicomios como factor básico para la condición de ciudadano de pleno derecho de los enfermos mentales. Cabe aquí recordar la constatación de la pérdida de garantías de los derechos fundamentales de los pacientes que residían en nuestros hospitales psiquiátricos.

\section{La disolución de los órganos directivos de la Salud Mental en el SAS}

La década de los ochenta estuvo, con sus dificultades y problemas, llena de luces. Sin embargo, no ocurre igual en la de los años noventa, deteniéndose el ritmo que la reforma había tenido en la década anterior. Los fondos económicos dedicados a la puesta en marcha de nuevos servicios sanitarios o en la mejora de los que ya estaban creados fueron prácticamente inexistentes, si exceptuamos la apertura de 7 comunidades terapéuticas en toda la década. Desde el SAS se cuestionaron los servicios de salud mental y las estructuras organizativas del IASAM fueron diluidas en los servicios centrales el SAS, quedando como servicio específico, uno denominado "Oficina de Salud Mental", servicio inédito hasta entonces con un nivel jerárquico muy bajo en los órganos de Dirección del SAS,. Esta dilución constituye un dato significativo en la actitud de la Administración Sanitaria Andaluza hacia el sistema de salud mental y de su concepción de la asistencia comunitaria.

A nuestro juicio, esta situación sobrevino a consecuencia de la complejidad que supuso el fenómeno de la integración de las estructuras de Salud Mental en el Sistema Sanitario General. Complicación que puede contemplarse desde dos perspectivas:

Las dificultades para la integración de lo mental en lo sanitario. La hegemonía de la asistencia hospitalaria en ese momento y nuestro sistema de enfoque comunitario y de organización mixta -al depender tanto de atención especializada (hospitales) como de AP (resto de profesionales de los ESMD) - hacía que fuéramos mal entendidos y por tanto rechazados. A estas alturas todavía no se ha resuelto el problema de esta doble dependencia en ninguno de los niveles de la organización.

Es curioso, por la contradicción que engendra, que todo el discurso actual del sistema sanitario general sobre la "orientación al ciudadano", "la continuidad de tratamientos y cuidados", "la cooperación interniveles", etc, son conceptos que, con parecidas denominaciones, hace tiempo que han sido asumidos por los sistemas de salud mental de todo el mundo. Lo que parece ocurrir es que no se tiene en cuenta todo lo que implican estos conceptos. Porque el que el ciudadano sea el centro del sistema, aplicado a la atención a la salud mental, significa dar a los pacientes psicóticos la condición plena de ciudadanos, como sujetos de plenos derechos, cosa que ha estado cuestionada hasta hace bien poco, asilándolos en manicomios. Pero es que cuando se dice la continuidad de cuidados, se está aludiendo a la desaparición de 
las barreras entre la comunidad y las estructuras hospitalarias, y, por último, cuando se habla de la cooperación es imprescindible tener el modelo comunitario presente.

Visto el fenómeno desde otra perspectiva, pudiéramos decir que el sistema sanitario general ha estado (está) preparado para dolencias de etiología biológica conocida y de evolución aguda. Es evidente que lo mental requiere de un enfoque holístico que implica unos servicios de atención bio-psico-social y, además, de un soporte de larga duración, que es precisamente lo opuesto a la ordenación sanitaria tradicional.

Las dificultades para pasar de ser marginal, a ser un servicio sanitario normalizado. Aunque la situación nos llevó casi a vivir y desde luego a compartir con Freeman (1999) las inquietudes vertidas en su revisión de los 50 años de asistencia psiquiátrica en el Reino Unido, es preciso también reconocer que por nuestra parte, la de los profesionales, existía un gran desconocimiento de las estructuras y del estilo de funcionamiento del servicio andaluz de salud. Los profesionales de atención a la salud mental habíamos pasado de ser absolutamente marginales, recluidos principalmente en los hospitales psiquiátricos, a tener un estatus menos marginal, pero también diferente al resto del sistema sanitario mientras duró el IASAM. Puede decirse que los profesionales no pudieron asumir la enorme demanda que supuso la confluencia, en la red sanitaria general, de la atención a los trastornos graves con aquellos otros que la cobertura asistencial universal traía consigo: depresiones leves, trastornos adaptativos, trastornos de ansiedad, etc.

Muchos cambios de estilo diferentes para poder asimilarlos todos de una manera eficaz. Así, se produjo un movimiento de repliegue hacia los nuevos dispositivos, desapareciendo la participación que había existido hasta entonces en el proceso de reforma. Esto también trajo consigo la desilusión y el escepticismo de muchos profesionales, que vieron como el modelo inicial con el que habían estado comprometidos se modificaba sustancialmente, por falta de recursos, ausencia de apoyo institucional e inexistencia de un movimiento organizado de profesionales suficientemente fuerte para que se pudiera continuar desarrollando el modelo comunitario. Las iniciativas en éste sentido, fueron escasas, minoritarias e ineficaces.

Probablemente la empresa de la integración era demasiado complicada como para haberla resuelto en breve espacio de tiempo. En resumen, son demasiados cambios a distintos niveles. Por un lado pasar, en un nivel de macrogestión, desde la beneficencia a un modelo sanitario público y universal; en el nivel asistencial de meso gestión, desde lo manicomial a lo comunitario; $y$, por último, de un modelo estrictamente biológico a otro bio-psico-social, en el que se ha avanzado poco y en el que otros intereses tomaron la delantera. De ahí la necesidad imperante de desarrollos de nuevos modelos integrativos que puedan ofrecer respuestas no a lo sintomatológico - dicho sea de paso, degeneración del modelo médico (Menéndez, 2005) sino a una psicopatología del sujeto y a unos abordajes integrados.

\section{Algunos datos asistenciales significativos de la década.}

Mientras que las dificultades expresadas en el apartado anterior no se despejaban, la actividad asistencial continuaba afrontando un incremento de la demanda. Estudios de finales de la década (Lehtinen, Riikonen, \& Lahtinen, 2000) muestran la importancia epidemiológica de los trastornos mentales en su conjunto. Así, entre un 20 y un $30 \%$ de la población general presenta trastornos mentales a lo largo de la vida. La depresión aparece como la segunda causa asociada a discapacidad según estimaciones para el año 2020. Se comentan a continuación algunos datos asistenciales de dispositivos en esta etapa.

En los ESMD se incrementó en un 26\% en cuanto al total de consultas, con mayor aumento de las primeras $(39,1 \%)$ que de las sucesivas (24\%). Lo que implicó que se disminuyera el índice sucesivas/primeras en un 9,4\%. Esto es un dato negativo para un servicio que está diseñado para realizar tratamientos especializados y de larga duración y que, por tanto, se debería tender a estabilizar la atención de las primeras consultas, mediante intervenciones de cooperación con los servicios de atención primaria, y ofrecer una relación sucesivas/primeras más óptima, sobre todo en los trastornos graves.

En relación con las unidades de hospitalización, los ingresos se incrementaron un 73,1\% en el periodo, mientras que el número de camas prácticamente se mantuvo, pues solo se registra un $5,6 \%$ de aumento, lo que equivale a la apertura de una nueva unidad. Sin embargo, no creemos que existiera un marcado déficit en cuanto al número de camas disponibles de corta estancia, sino más bien que el aumento de ingresos se deba, por un lado, a la escasa dotación de servicios sociocomunitarios en gran parte de este periodo y, por otro, a un modelo de intervención que solo parcialmente contemplaba el abordaje psicosocial dentro de una amplia visión de redes comunitarias, por lo que a menor soporte, mayor número de ingresos.

Más preocupante, a nuestro juicio, es el descenso de la estancia media en un $23,7 \%$ en todo el periodo, una manifestación más de la tendencia al mimetismo con un modelo de intervención médico-quirúrgico, tanto en lo clínico ("lavado" rápido de síntomas), cuanto en la gestión (más eficiencia con menor estancia media). Uno y otro caso son engañosos; el primero por lo que implica de aumento de reingresos si solo se atiende a lo sintomatológico y no a una visión psicopatológica global; el segundo porque no está claro el beneficio, por lo que acabamos de decir, sino tampoco el coste: el coste de estas unidades no está tanto en función de las estancias, al ser unidades cerradas y soportar los costes sobre todo en los recursos humanos asignados, que son fijos, y no en los costes de los procesos asistenciales (pruebas complementarias, fármacos, etc.) que no son tan altos si lo comparamos con otros servicios.

Los datos de las USMIs revelan también un crecimiento muy notable de la demanda asistida, un 58,2\% respecto de las primeras consultas y casi el doble de las sucesivas. Sin embargo, la relación sucesivas/primeras no solo no se resintió, sino que aumentó hasta un $24,7 \%$, lo que denota una optimización de la capacidad asistencial en este tipo de dispositivo ya en la década a la que nos estamos refiriendo. Hay que tener en cuenta que todavía no se habían reforzado los mismos con hospitales de día, cuestión que se abordó en la siguiente etapa.

Para finalizar este apartado, hay que concluir que, en efecto, se cerraron los Hospitales Psiquiátricos, como hemos dicho, pero 
que, una vez externalizada la población geriátrica, de deficientes, trastornos orgánicos y menos de psicosis residuales - lo que ocurrió en la primera mitad de los años noventa (López, Benítez, García, \& Weisman, 1998) - quedaba una población más difícil de deshospitalizar, atender en la comunidad y de hacerla menos dependiente mediante su reinserción en la sociedad. Pues bien, la mayoría de los pacientes que quedaban en los hospitales psiquiátricos fueron trasladados a las comunidades terapéuticas, lo que teniendo en cuenta sus dotaciones y, por otra parte, la escasa implantación de los recursos sociales que después aporta FAISEM, provocó cierto estancamiento y confusión entre los espacios estrictamente sanitarios y los sociales.

\section{Etapa de la salud mental reformada: 2001-2011}

Salud mental y contexto político y social

En el año 2000 se cierra el último hospital psiquiátrico de Andalucía que permanecía abierto, Miraflores en Sevilla. Pero por otro lado, a pesar de la puesta en marcha de recursos destinados fundamentalmente a los trastornos mentales graves (comunidades terapéuticas y FAISEM), se constata una insuficiencia de recursos. Este déficit está derivado de varias circunstancias de las que destacamos dos: el dimensionamiento de los recursos necesarios previsto en 1984, partió de un desconocimiento lógico de las necesidades asistenciales reales a atender, y del incremento progresivo de la demanda ocasionado por la mejora de la accesibilidad y por los cambios sociales que se producen en la sociedad del bienestar.

Esta situación da lugar a momentos de cierto nivel de conflicto con varios protagonistas: las asociaciones de familiares, generalmente integradas por personas afectadas por problemas derivados de la convivencia con personas que padecen un Trastorno Mental Grave, o Trastornos de la Conducta Alimentaria, la Asociación Andaluza de Neuropsiquiatría (Asociación de Profesionales de Salud Mental) y la propia Administración Sanitaria.

Cada protagonista tiene sus propios argumentos para sostener su posición. De esta manera:

Las asociaciones de familiares sostienen que existe una falta de recursos para la atención a los trastornos mentales graves (rehabilitación y medias y largas estancias), infraestructuras deficientes en las unidades de hospitalización de los hospitales generales o escasa atención domiciliaria y atención y apoyo a familiares. En otro orden, demandas de recursos específicos para el fenómeno emergente de los trastornos alimentarios.

Los profesionales de salud mental, estando de acuerdo en muchas cosas con las asociaciones de familiares, pone el énfasis en los déficit que atañen a la organización y dimensionamiento de los servicios, a lo relacionado con la calidad asistencial y en los cambios cuantitativos y cualitativos habidos en la demanda. Resultado de su preocupación y reflexiones puede considerarse el Informe sobre la Salud Mental de Andalucía, publicado en 2003 por la Asociación Andaluza de Neuropsiquiatría (Asociación Andaluza de Neuropsiquiatría de Profesionales de la Salud Mental, 2003).

La administración sanitaria, desde el marco del Plan de Calidad elaborado por la Consejería de Salud, realizó un esfuerzo para comprender éstas demandas, reflexionando sobre ellas, y estableciendo unos cauces de diálogo, con los agentes citados. Sin embargo, al disponer de escasos indicadores sobre los que evaluar el rendimiento de los recursos, y menos aún los resultados, tiene dificultades para realizar una planificación acertada.

En este escenario se inicia desde nuestro punto de vista una nueva etapa, la de "la Salud Mental Reformada". No existió una clausura oficial del proceso de reforma, pero creemos que este proceso debe darse por terminado por dos razones fundamentalmente:

1 - Los objetivos que definió el IASAM para la reforma se han cumplido: el cierre de los hospitales psiquiátricos, la creación de una red de servicios alternativos y un modelo asistencial basado en los presupuestos de la Psiquiatría Comunitaria.

2 - Existe una nueva etapa derivada, fundamentalmente, de las nuevas necesidades de atención emergidas a lo largo de estos años y, por otra parte, de las nuevas orientaciones en la gestión de los servicios sanitarios públicos existentes en la actualidad.

Por otra parte, otro aspecto que define esta etapa desde el contexto de los servicios públicos de salud de nuestra autonomía es el Plan de Calidad, elaborado por la Consejería de Salud del Gobierno Autonómico Andaluz, y apoyado en dos objetivos:

- La Atención por Procesos Asistenciales Integrados, con el objetivo de prestar una atención de calidad basada en la evidencia y garantizando la continuidad asistencial.

- Estimular una organización de los Servicios basados en Unidades de Gestión Clínica.

Ambos objetivos tienen como elementos comunes la consideración del usuario como centro del sistema sanitario y la participación de los profesionales.

Este Plan de Calidad tiene su impacto sobre el subsistema de atención a la Salud Mental, elaborándose los siguientes procesos relacionados con trastornos mentales: a) el Proceso Ansiedad, Depresión y Somatizaciones, b) el Proceso de los Trastornos de la Conducta Alimentaria, y c) el Proceso del Trastorno Mental Grave. Y, por otra parte, se estimula desde la Organización Sanitaria la creación de Unidades de Gestión Clínica de Salud Mental.

\section{Los Planes Integrales de Salud Mental}

Tanto el Plan Integral de Salud Mental 2004-2007 cuanto el II Plan 2008-2011 enmarcan la etapa actual de desarrollo de la atención a la salud mental. Suponen un nuevo impulso de atención a la salud mental desde el Sistema Sanitario Público de Andalucía en el contexto de calidad citado. El primero de los planes definió los siguientes objetivos:

1. Aumentar el grado de conocimiento e información sobre la salud mental, de las personas con trastorno mental, su entorno cercano y de la sociedad andaluza.

2. Mejorar la calidad de vida de las personas con trastornos mentales y sus allegados.

3. Adecuar la oferta de servicios a las necesidades de la población, efectiva y eficiente.

4. Garantizar a las personas con trastornos mentales una atención sanitaria basada en el enfoque comunitario y la estructuración del proceso asistencial desde la perspectiva de la continuidad de cuidados como elemento de calidad integral. 
5. Potenciar el conocimiento - formación e investigación de los profesionales sobre los factores de riesgo y las estrategias de intervención, para potenciar la toma de decisiones clínicas basadas en la evidencia. Algunos datos de esta etapa figuran en las tablas siguientes.

Como puede observarse en la tabla 2, la demanda atendida en las unidades comunitarias se ha estabilizando - el \% de variación ha sido solo de 1,49 en tres años - producto, por un lado, del incremento de recursos en estos últimos años, tanto en el ámbito sanitario como en el social, y, por otro, de la puesta en marcha de modelos de cooperación con los centros de atención primaria. La tendencia, en estos momentos continúa, con lo que

Tabla 2

Evolución de las personas atendidas en unidades de Salud Mental Comunitaria según grupo diagnóstico. Tasas por 100.000 habitantes. SSPA, 2003-2006.

\begin{tabular}{llrrrrr}
\hline \multirow{2}{*}{ CIE-10 } & \multicolumn{1}{c}{ Grupo diagnóstico } & Año 2003 & Año 2004 & Año 2005 & Año 2006 & $\begin{array}{r}\text { \% variación } \\
20003-2006\end{array}$ \\
\hline F00-F09 & T. orgánicos & 44,79 & 48,46 & 41,99 & 46,50 & 3,82 \\
F10-F19 & T. consumo sustâncias & 32,81 & 35,84 & 35,06 & 38,76 & 18,20 \\
& psicotropas & & & & \\
F20-F29 & T. esquizofrénicos e ideas & 223,72 & 239,23 & 236,35 & 256,08 & 14,46 \\
& delirantes & 482,05 & 502,17 & 483,27 & 517,66 & 7,39 \\
F30-F39 & T. humor & 668,28 & 747,37 & 728,69 & 815,58 & 22,04 \\
F40-F49 & T. neuróticos & 37,57 & 40,88 & 35,72 & 37,16 & $-1,09$ \\
F50-F59 & Disfunciones fisiológicas y & & & & & 34,67 \\
& factores somáticos & 70,46 & 89,60 & 83,39 & 94,89 & 14,53 \\
F60-F69 & T. personalidad & 39,99 & 42,25 & 42,71 & 45,80 & 36,70 \\
F70-F79 & Retraso mental & 5,94 & 6,59 & 6,41 & 8,12 & 11,97 \\
F80-F89 & T. desarrollo & 64,18 & 67,18 & 62,89 & 71,86 & $-32,78$ \\
F90-F98 & T. infancia y adolescência & 767,74 & 684,41 & 648,81 & 516,04 & 127,13 \\
F99 & T. mental sin especificación & 20,27 & 32,41 & 33,58 & 46,04 & 1,49 \\
Total & Sin patologia psiquiátrica & $2.457,81$ & $2.536,37$ & $2.438,88$ & $2.494,52$ & \\
\hline
\end{tabular}

Nota. Fuentes: Sistema de información de salud mental de Andalucía. Revisión del Padrón Municipal de

Habitantes a 1 de enero de 2003 y 2006. Instituto de Estadística de Andalucía.

se podría ir logrando un objetivo que hace una década parecía inalcanzable y no es otro que nuestros ESMD puedan realizar intervenciones especializadas contando con tiempo adecuado sin que se colapsen por una demanda indiscriminada.

Referente las hospitalizaciones breves, la figura 3 muestra indicadores estables en los ingresos de las unidades de hospitalización siendo, como es lógico la carga asistencial mucho mayor en el GDR 430 (psicosis) para el que se obtienen una estancia media más acorde con nuestra realidad clínica. Es de señalar que el índice de ocupación en estas unidades se ha normalizado en todo el territorio, lo que se debe en gran medida al desarrollo de otros tipos de dispositivos que han evitado la residencialización de pacientes en ellas.

Las tablas 3, 4 y 5, ofrecen la evolución en los últimos años de la actividad asistencial de CT, HD y URA. En todos ellas la actividad ha sufrido un aumento considerable con $\%$ de variación marcadamente altos, lo que se corresponde con el aumento de recursos - tanto de profesionales, como de dispositivos- que se ha operado en estos años

Finalmente en las dos siguientes tablas (6 y 7) se expone el desarrollo de servicios que ha arrojado la implantación del PISMA I. Como puede observarse, en el ámbito sanitario, destaca el incremento de recursos referidos a la población infantojuvenil, las plazas de media-larga estancia (CT) y la oferta de plazas en Hospitales de Día. Por otra parte, el incremento de recursos sociales destinados a personas con trastorno mental grave ha sido notable, destacando los \% de variación en relación con los programas residenciales (más de 300 plazas en Casas-Hogares, 400 plazas en viviendas supervisadas) y con el programa ocupacional/laboral, especialmente en este último, la orientación y la integración laboral en empresas sociales. Bien es cierto que la crisis económica ha frenado el crecimiento en los dos últimos años.

En relación con la mejora de los procedimientos también es de destacar la gestión sanitaria a través de los procesos asistenciales integrados, que tratan de evitar la variabilidad de la práctica clínica. En nuestro entorno se definieron e implantaron en estos años tres procesos asistenciales:

Proceso Ansiedad, Depresión y Somatizaciones (Consejería de Salud de la Junta de Andalucía, 2002). Dirigido a Atención Primaria y los Servicios de Salud Mental. Centrado en el trabajo de interfase, desarrollando espacios de comunicación y cooperación entre ambos, con la finalidad de incrementar los servicios ofertados a los pacientes con estos tipos de trastornos a través de un uso más adecuado y eficiente de los recursos.

Proceso Trastorno Mental Grave (Consejería de Salud de la Junta de Andalucía, 2006). Dirigido a la Atención Primaria y los Servicios de Salud Mental. Centrado en la atención de aquellos pacientes que presentan varias de las siguientes características: sintomatología de carácter psicótico, evolución crónica, con graves distorsiones en sus relaciones interpersonales, posible riesgo para su vida, déficit en su funcionamiento cotidiano y con la necesidad de intervenciones complejas en su atención sanitaria y/o social.

Proceso Trastornos de la Conducta Alimentaria (Consejería de Salud de la Junta de Andalucía, 2004). Dirigido así mismo a garantizar una continuidad terapéutica de los sujetos con estos 


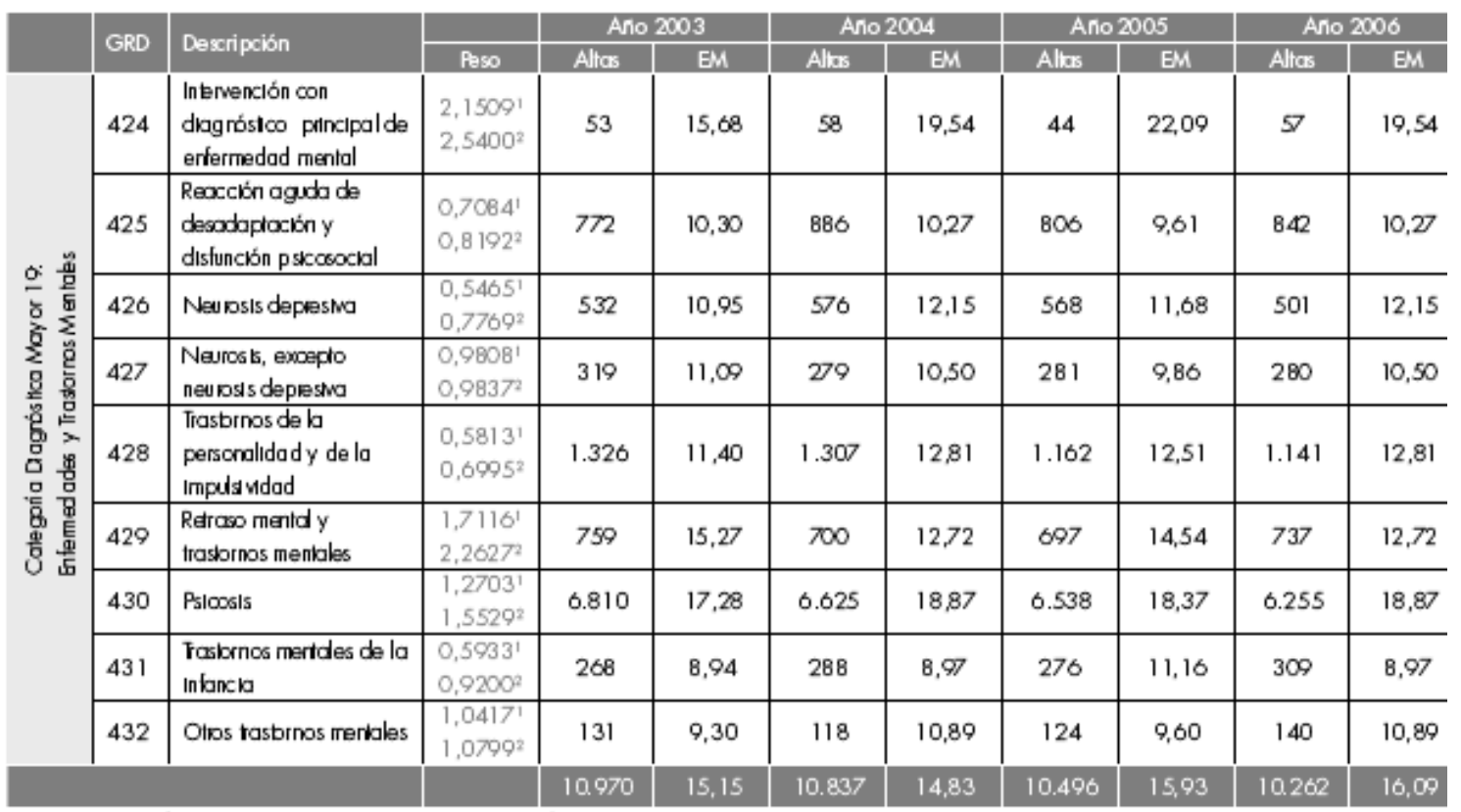

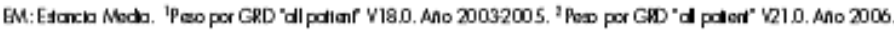

Fuste: Corpurbo Mhino Básto do Dalos d Ala Hospldab, 20002006. Servico Ardduz de Sadud.

Figura 3. Altas hospitalarias por enfermidades y transtornos mentales según GRD. SSPA, 2003-2006 Nota. Fuente: Servicio Andaluz de Salud.

Tabla 3

Evolución de la actividad asistencial de las comunidades terapéuticas de salud mental. SSPA, 2003-2006

\begin{tabular}{lccccc}
\hline Actividad asistencial & Anõ 2003 & Año 2004 & Año 2005 & Año 2006 & $\begin{array}{c}\text { \% variación } \\
\text { 2003-2006 }\end{array}$ \\
\hline Hospitalización completa & & & & & \\
Pacientes & 319 & 314 & 325 & 351 & 10,03 \\
Estancias & 60.947 & 62.682 & 69.743 & 70.735 & 16,05 \\
$\begin{array}{l}\text { Programa de Día } \\
\text { Pacientes }\end{array}$ & 392 & 430 & 484 & 557 & 42,09 \\
Estancias & 32.125 & 34.683 & 36.502 & 39.483 & 22,90 \\
\hline
\end{tabular}

Nota. Fuente: Programa de Salud Mental. Dirección General de Asistencia Sanitaria. Servicio Andaluz de Salud.

Tabla 4

Evolución de la actividad asistencial de los hospitales de día de salud mental. SSPA, 20032006

\begin{tabular}{lccccc}
\hline Actividad asistencial & Anõ 2003 & Año 2004 & Año 2005 & Año 2006 & $\begin{array}{c}\text { \% variación } \\
\text { 2003-2006 }\end{array}$ \\
\hline Ingresos & 523 & 681 & 751 & 762 & 45,68 \\
Altas & 298 & 372 & 451 & 557 & 86,91 \\
Pacientes & 1.045 & 1.081 & 1.391 & 1.596 & 52,72 \\
Estancias & 22.878 & 32.771 & 35.973 & 44.758 & 60,54 \\
\hline
\end{tabular}

Nota. Fuente: Programa de Salud Mental. Dirección General de Asistencia Sanitaria. Servicio Andaluz de Salud. 
Tabla 5

Evolución de la actividad asistencial de las unidades de rehabilitación de salud mental. SSPA, 2003-2006

\begin{tabular}{lccccc}
\hline Actividad asistencial & Año 2003 & Año 2004 & Año 2005 & Año 2006 & $\begin{array}{c}\text { \% variación } \\
2003-2006\end{array}$ \\
\hline Ingresos & 405 & 670 & 533 & 446 & 10,12 \\
Altas & 159 & 228 & 328 & 330 & 107,54 \\
Pacientes & 1.552 & 1.678 & 1.830 & 1.946 & 25,38 \\
Estancias & 65.253 & 78.350 & 81.112 & 87.322 & 33,82 \\
\hline
\end{tabular}

Tabla 6

Evolución de los dispositivos de salud mental en el SSPA durante el periodo de vigencia del i pisma. 2003-2007 Dispositivos USMC

Se han creado 4 nuevas USMC, Incremento 03-07

USMC dos de las cuales corresponden a desdoblameintos de unidades ya

USMI-J existentes.

Se há creado uma nueva USMI-J al De 13 a 14 dispositivos, de 11 a 39 desboblarse uma ya existente, outra há sido reformada y se han habilitado 28 camas y creado 252 plazas de hospital de día para infancia y adolescência.

UHSM

CTSM

URSM

HDSM
Se han reformado 6 unidades de hospitalización, com 25 nuevas camas.

Se ha abierto uma nueva CTSM, 4 han sido reformados y se há incrementado el $\mathrm{n}^{\circ} \mathrm{de}$ camas en vários de los dispositivos, creándose 41 nuevas camas. Se han reforzado 2 URSM, creándose 40 plazas de hospital de día.

Se han aberto 6 nuevos hospitales de día, com 120 plazas y há reformado uno de los existentes. camas y de 0 a 252 plazas de hospital de día para infancia y adolescência.

De 72 a 76 dispositivos

De 523 a 548 camas em unidades de hospitalización.

De 13 a 14 dispositivos y 189 a 230 camas em comunidades terapêuticas.

De 0 a 40 plazas de hospital de día em unidades de reabilitación.

De 5 a 11 dispositivos y de 100 a 220 plazas de hospital de día de personas adultas.

Tabla 7

Evolución de los recursos de apoyo social para personas com transtorno mental grave. FAISEM, 2003-2007

\begin{tabular}{|c|c|c|c|c|c|c|c|c|c|c|c|}
\hline \multirow{2}{*}{ Recursos FAISEM } & \multicolumn{5}{|c|}{ Año 2003} & \multicolumn{5}{|c|}{ Año 2007} & \multirow{2}{*}{$\begin{array}{c}\% \\
\text { Variación } \\
2003-2007 \\
\text { plazas x } \\
100.000 \\
\text { hab. }\end{array}$} \\
\hline & $\begin{array}{l}\mathrm{N}^{\circ} \\
\text { Disp. }\end{array}$ & $\begin{array}{c}\mathrm{N}^{\circ} \\
\text { Plazas }\end{array}$ & $\begin{array}{c}\text { Plazas X } \\
100.000 \\
\text { hab. }\end{array}$ & $\begin{array}{c}\% \\
\text { Hombres }\end{array}$ & $\begin{array}{c}\% \\
\text { Mujeres }\end{array}$ & $\begin{array}{l}\mathrm{N}^{\circ} \\
\text { Disp. }\end{array}$ & $\begin{array}{c}\mathrm{N}^{\circ} \\
\text { Plazas }\end{array}$ & $\begin{array}{c}\text { Plazas X } \\
100.000 \\
\text { hab. }\end{array}$ & $\begin{array}{c}\% \\
\text { Hombres }\end{array}$ & $\begin{array}{c}\% \\
\text { Mujeres }\end{array}$ & \\
\hline \multicolumn{12}{|l|}{ Programa residencial } \\
\hline Casas-Hogar & 22 & 335 & 4,4 & 69,2 & 30,8 & 47 & 742 & 9,2 & 68,8 & 31,2 & 109,1 \\
\hline $\begin{array}{l}\text { Viviendas } \\
\text { supervisadas }\end{array}$ & 94 & 348 & 4,6 & 78,3 & 21,7 & 175 & 667 & 8,3 & 73,6 & 26,4 & 80,4 \\
\hline $\begin{array}{l}\text { Plazas en } \\
\text { residencias }\end{array}$ & 39 & 216 & 2,8 & 66,2 & 33,8 & 32 & 151 & 1,9 & 70,4 & 29,6 & 32,1 \\
\hline $\begin{array}{l}\text { Plazas en } \\
\text { pensiones }\end{array}$ & 5 & 12 & 0,2 & 71,4 & 28,6 & 2 & 12 & 0,1 & 72,2 & 27,8 & $-50,0$ \\
\hline $\begin{array}{l}\text { Atención } \\
\text { domiciliaria }\end{array}$ & & 119 & 1,6 & 67,6 & 32,2 & & 410 & 5,1 & 61,2 & 38,8 & 218,8 \\
\hline \multicolumn{12}{|c|}{ Programa ocupacional/laboral } \\
\hline $\begin{array}{l}\text { Talleres } \\
\text { ocupacionales }\end{array}$ & 94 & 1.647 & 21,7 & 76,6 & 23,4 & 115 & 2.251 & 27,9 & 73,1 & 26,9 & 28,6 \\
\hline Cursos F.P.O. & 60 & 302 & 4,0 & 76,8 & 23,2 & 53 & 261 & 3,2 & 72,1 & 27,9 & 20,0 \\
\hline Empresas sociales & 9 & $189^{\mathrm{a}}$ & 2,5 & 79,6 & 20,4 & 9 & $225^{\mathrm{a}}$ & 2,8 & 76,2 & 23,8 & 12,7 \\
\hline $\operatorname{SOAE}^{\mathrm{b}}$ & 8 & $134^{\mathrm{c}}$ & 1,8 & 68,6 & 31,4 & 8 & $952^{\mathrm{C}}$ & 11,8 & 67,5 & 32,5 & 569,9 \\
\hline \multicolumn{12}{|c|}{ Programa ócio y tempo libre } \\
\hline Clubes sociales & 28 & 840 & 11,0 & 75,2 & 24,8 & 45 & 1.407 & 17,5 & 70,7 & 29,3 & 59,1 \\
\hline $\begin{array}{l}\text { Programa da } \\
\text { vacaciones }\end{array}$ & & 885 & 11,6 & 74,3 & 257 & & 823 & 10,2 & 67,8 & 32,2 & 12,1 \\
\hline \multicolumn{12}{|l|}{ Programa Tutelar } \\
\hline Entedades tutelares & 6 & 542 & 7,1 & 71,3 & 28,7 & 8 & 655 & 8,1 & 70,1 & 29,9 & 14,1 \\
\hline \multicolumn{12}{|c|}{ Programa atención a personas com TMG sin hogar } \\
\hline Plazas & & & & & & & 37 & 0,5 & 74,8 & 25,2 & \\
\hline
\end{tabular}

Nota. Fuentes: Fundación Pública Andaluza para la Integración Social de Personas com Enfermedad Mental.

Revisión del Padron Municipal de Habitantes a 1 de enero de 2003 y 2007, Instituto de Estadística de Andalucía.

${ }^{a}$ Personas trabajadoras com enfermedad mental.

${ }^{\mathrm{b}}$ Servicios de Orientación y Apoyo al Empleo.

${ }^{\mathrm{c}} \mathrm{N}^{\mathrm{o}}$ de contratos. 
trastornos desde la Atención Primaria hasta las necesidades de tratamiento especializado que requieran: Salud Mental, Medicina Interna, Endocrino, etc.

Otra de las facetas en la que se ha producido una evolución que merece señalar se refiere a la estructura organizativa. Para ello se promulgó un nuevo decreto de ordenación administrativa y funcional de los servicios de salud mental. Supone la organización de los mismos en Unidades de Gestión Clínica, lo que, de forma resumida, implica el acuerdo de gestión como marco de gestión de la Unidad. Lo que a su vez lleva aparejada la evaluación de cada profesional y la asignación de incentivos económicos; todos los dispositivos y profesionales de salud mental estarán adscritos a la atención especializada; dirección de la unidad seleccionada por concurso público; todos los profesionales estarán vinculados jerárquica y funcionalmente al director/a de la unidad, que contará con el apoyo en las labores de dirección del/la coordinador/a de cuidados de enfermería y de los coordinadores/as de dispositivos asistenciales; Equipos multidisciplinares; y.identificación de las categorías profesionales que integrarán los dispositivos.

El PISMA II, actualmente en vigor, trata de profundizar en la implantación de recursos, procedimientos y organización emprendidos en el primer PISMA, si bien en lo referente a recursos el impasse es la regla debido a los tiempos de restricción económica. Sí es de destacar la atención que se pretende a aspectos de la salud mental novedosos en nuestro medio, como son: a) Detección e intervención precoz de los trastornos psicóticos; b) Tratamiento intensivo en la comunidad a personas con trastorno mental grave; c) Atención específica a personas con trastornos de personalidad; d) Atención a la salud mental a personas en riesgo o situación de exclusión social; e) Perspectiva de género en todas las líneas de intervención.

Estos aspectos junto con la continuidad de los recogidos en el primer plan, conforman 15 líneas estratégicas de intervención con las que se pretende continuar el desarrollo iniciado en los primeros años del milenio.

Desde otra perspectiva más amplia de la salud mental y de la salud en general hay que contemplar lo que supone la Ley de Promoción de la Autonomía Personal y Atención a las Personas en Situación de Dependencia. En estos momentos es uno de los escenarios más importantes pues supone la universalización progresiva, prevista hasta el año 2015, de los servicios sociales destinados al fomento de la autonomía en personas dependientes, incluyendo a las personas con trastorno mental grave. En consecuencia, dichas personas tendrán derecho a una serie de prestaciones: centros de día, ayuda a domicilio, plazas residenciales, prestación económica a cuidadoras, etc., que son valoradas y asignadas conjuntamente por profesionales de ambos entornos:

\section{Perspectiva actual de la salud mental en Andalucía}

A nadie se le escapa que si hemos de destacar un aspecto del proceso de reforma de la salud mental en nuestra Comunidad Autónoma es la desaparición física de los ocho Hospitales Psiquiátricos provinciales y su sustitución por una red alternativa. Todo este proceso, complejo y no exento de dificultades de todo tipo, se ha llevado a cabo por el esfuerzo individual y colectivo de muchos protagonistas a lo largo de estos años. Los Poderes Públicos, la Administración Sanitaria, las Asociaciones Profesionales, las Asociaciones de Familiares de Enfermos Mentales. A pesar de desacuerdos, conflictos y dudas de los diferentes agentes e instituciones que han intervenido, ha habido un hilo conductor de las transformaciones a la que hemos asistido en estos años: la recuperación de la dignidad de las personas con trastornos mentales y la modernización de los medios a emplear en su atención.

Todas las transformaciones señaladas, han dado solución a problemas de la asistencia en salud mental en Andalucía, pero, no solo han surgido otros nuevos, sino que algunos de "los de siempre" quedan por resolver. Parte de estos problemas son de orden interno y se refieren al modo en el que se ha llevado a cabo la reforma o cómo se ha orientado la reflexión sobre ella (Fernández Liria, 1997), haciendo más hincapié en la creación de nuevos dispositivos de atención, que en la reflexión y el análisis, en la calidad de las actuaciones o en la disminución de la variabilidad. Por eso, queremos finalizar con algunas consideraciones sobre las necesidades actuales y los retos de futuro de la salud mental de Andalucía:

Hemos señalado como finalizada la etapa de Reforma de la Salud Mental. Sin embargo, en nuestra Comunidad Autónoma existen aún 163 camas hospitalarias "no reformadas" dependientes de la Administración de Justicia en el Hospital Penitenciario de Sevilla. Sería necesario buscar una solución acordes con los principios y las técnicas actuales de atención a la salud mental para la población reclusa que presenta problemas de salud mental.

Otro problema estructural pendiente de solución es el de la integración de la atención a los problemas derivados del consumo de sustancias tóxicas en el Sistema Sanitario Público Andaluz. De tal manera, que los ciudadanos con este tipo de trastornos dispongan de servicios homologados y en las mismas condiciones en cuanto a derechos y deberes que para el resto de los problemas de salud.

Algunas infraestructuras tienen problemas de habitabilidad o sus dimensiones o condiciones no se encuentran acordes con los objetivos terapéuticos a los que deberían estar subordinados.

Existe, como en el resto de las especialidades el problema de la variabilidad de las prestaciones asistenciales y de sus resultados. Desde el análisis de los indicadores de los dispositivos, las comunicaciones y ponencias en jornadas y congresos se advierte esta variabilidad en dispositivos semejantes o entre zonas geográficas distintas. Así, encontramos diferencias en la atención comunitaria de los pacientes con trastornos mentales graves, en el modo de relación y gestión de la demanda derivada desde los dispositivos de atención primaria, el modo como se gestiona y atiende en las unidades de salud mental de estancias cortas de los hospitales generales, en el modo de abordar la rehabilitación o la gestión de las comunidades terapéuticas y la gestión de los recursos orientados a la reinserción social y laboral de los pacientes mentales, o la atención a los problemas de la infancia o adolescencia, etc.

Una de las claves que nos hace situarnos en otra etapa totalmente distinta es la transformación de la demanda en los últimos años. Socialmente se ha pasado de la "negación- 
ocultación" de los problemas de salud mental a la psicologízación de cada dificultad de la vida cotidiana. Estos problemas de la vida cotidiana, al llegar a los servicios especializados restan recursos a aquellos trastornos que sí los requieren, dado que suponen un incremento muy importante de la demanda, tanto en Salud Mental como en Atención Primaria.

La otra característica en la transformación de la demanda es la emergencia de otros problemas de salud mental que con antelación y como consecuencia de la falta de servicios estaban ocultos. En esta categoría se encuentran los problemas de la infancia y adolescencia y la población geriátrica. A estos problemas hay que añadir los que empiezan a surgir como los relacionados con la población inmigrante. Son los nuevos retos a los que hay que hacer frente de forma inmediata.

Otro reto importante, aunque no nuevo, es el de la formación de los profesionales. Los nuevos retos asistenciales de los que hemos hablado y las nuevas formas de gestión sanitaria la requieren. En este sentido es necesario un esfuerzo importante entre todos: la Administración sanitaria y las asociaciones científicas. La actualización de conocimientos de los profesionales de la salud mental y de la atención primaria, entre otros, es imprescindible si queremos una atención de calidad que de respuesta a las necesidades de los pacientes.

Por último, vamos a incidir en un aspecto que creemos de extraordinaria magnitud. Nos referimos a la importancia y trascendencia del concepto sujeto psico(pato)lógico. La noción que los profesionales de la salud mental tengan sobre este concepto, aunque también los de otros campos de la salud o en general de los servicios públicos, incidirá directamente en la asistencia tanto a pacientes como a familiares. Si no tenemos en nuestro esquema referencial un modelo de sujeto psicológicorelacional y ciudadano podemos correr el riesgo de desandar lo recorrido. El camino de conformación de nuevas fórmulas para la marginación no es difícil, sobre todo en una sociedad en la que no predominan precisamente valores como el esfuerzo continuado y la solidaridad; tendiendo más bien al hedonismo, la inhibición social y a soluciones "económicamente rentables".

Esta es una responsabilidad que atañe de manera muy especial a los profesionales pero también a las Instituciones Públicas, que son las que planifican y ejecutan.

Esta consideración de la persona como sujeto, ha sido determinante para favorecer el proceso de reforma que hemos vivido, pero también lo es para el futuro de nuestro modelo de servicios donde, no lo olvidemos, el peligro de regresión a formas de trabajo manicomiales, con o sin manicomios, está siempre está presente. No viene mal recordar algunas de las reflexiones que nos transmitió el sociólogo francés Robert Castel (1984), a propósito de sus análisis sobre los procesos de reformas psiquiátricas en EEUU y Francia en los años 1960 y 1970 del siglo pasado y las dificultades y riesgos que llevan aparejadas; lo que nos obliga a una permanente reflexión sobre nuestro quehacer cotidiano y sus bases conceptuales y epistemológicas.

\section{Referencias}

Asociación Andaluza de Neuropsiquiatría de Profesionales de la Salud Mental (2003). Informe La Salud Mental en Andalucía. Cuadernos Técnicos, 1(9).

Castel, C. (1984). La gestión de los riesgos. Barcelona: Anagrama.

Consejería de Salud de la Junta de Andalucía (2002). Ansiedad, depresión, somatizaciones: proceso asistencial integrado. Sevilla: Consejería de Salud de la Junta de Andalucía.

Consejería de Salud de la Junta de Andalucía (2004). Trastornos de la conducta alimentaria: proceso asistencial integrado. Sevilla: Consejería de Salud de la Junta de Andalucía.

Consejería de Salud de la Junta de Andalucía (2006). Trastorno mental grave: proceso asistencial integrado. Sevilla: Consejería de Salud de la Junta de Andalucía.

Consejería del Salud de la Junta de Andalucía (2008). II Plan Integral de Salud Mental de Andalucía (2008-2012). Sevilla: Servicio Andaluz de Salud.

Servicio Andaluz de Salud (2003). Plan Integral de Salud Mental de Andalucia (2003-2007). Junta de Andalucía: Biblioteca del Servicio Andaluz de Salud.

Fernández Liria, A. (1997). La reforma psiquiátrica en España: en qué (y en qué no) metimos la pata. Psiquiatría Pública, 9(15), 167-175.

Freeman, H. L., Fryers, T., \& Henrderson, J. H. (1985). Mental health services in Europe: 10 years on. Copenhagen: World Health Organization Regional Office for Europe.

Freeman, H. (1999). Psychiatry in the National Health Service. British Journal of Psychiatry, 175, 3-11.

Lehtinen, V., Riikonen, E., \& Lahtinen, E. (2000). Promotion of Mental Health on the European Agenda (Relatório Técnico). Recuperado de http://groups. stakes.fi/NR/rdonlyres/F744090C-6539-484D-A87A-36BFDFE0CACE/0/ Promotion.pdf

López, M., Benítez, F., García, M. J., \& Weisman, L. C. (1998). Un programa para la supresión de los hospitales psiquiátricos públicos en Andalucía (1985- 1995). In J. Garcia, A. Espino, \& L. Lara (Orgs.), La Psiquiatría en la España de fin de siglo (pp. 101-116). Madrid: Díaz de Santos.

Menéndez Osorio, F. (2005): Veinte años de la Reforma Psiquiátrica. Panorama del estado de la Psiquiatría en España de los años 1970 a los 2000: de un pensamiento único a otro. Revista de la Asociación Española de Neuropsiquiatría, 25(95), 69-81

Torres González, F. (Org.). (1999). Red Maristan. Cuidados y necesidades de las personas afectadas por trastornos esquizofrénicos que viven en la comunidad. Documento para el Consenso Internacional. Granada: Red Maristan. 
José Carmona Calvo é psiquiatra, coordinador da Unidad de Salud Mental de Jerez, Unidad de Gestión Clínica de Salud Mental del Hospital General de Jerez. Servicio Andaluz de Salud. Endereço para correspondencia: C./ José Luis Díez no 14, $5^{\text {a }}$ planta. Jerez de la Frontera, 11403. Cádiz, España. Telefones ++34 956-033627/ 628; Fax: ++34 956-033629. E-mail: jose.carmona.calvo.sspa@juntadeandalucia.es

Francisco del Río Noriega é psiquiatra, director de la Unidad de Gestión Clínica de Salud Mental del Hospital General de Jerez. Servicio Andaluz de Salud. E-mail: francisco.rio.sspa@juntadeandalucia.es 